\title{
Micro-CT Evaluation of Voids in the Filling Material of Single-Rooted Teeth Obturated with Different Techniques
}

\author{
Fatma Asheibi ${ }^{1,2}$, Alison J.E. Qualtrough ${ }^{1}$, Anthony Mellor ${ }^{1}$, Philip J. Withers ${ }^{3}$ \\ and Tristan Lowe ${ }^{3}$ \\ ${ }^{1}$ School of Dentistry, University of Manchester, Manchester, UK \\ ${ }^{2}$ Garyounis University, Faculty of Dentistry, Benghazi, Libya \\ ${ }^{3}$ Henry Moseley X-ray Imaging Facility, School of Materials, University of Manchester, Manchester, \\ UK
}

Correspondence should be addressed to: Fatma Asheibi; Fafadent1@yahoo.com

Received Date: 11 October 2013; Accepted Date: 8 January 2014; Published Date: 22 March 2014

Academic Editor: Mohammad M. Hammad

Copyright (C) 2014 Fatma Asheibi, Alison J.E. Qualtrough, Anthony Mellor, Philip J. Withers and Tristan Lowe. Distributed under Creative Commons CC-BY 3.0

\begin{abstract}
The aim of this study was to compare the volume of voids in a resin-based root canal filling (Resilon) with gutta percha using either cold lateral condensation or continuous wave of compaction filling techniques using computed X-ray micro-tomography (micro-CT).

A total of 56 extracted upper anterior human teeth were decoronated, the canals shaped using rotary ProTaper files to a final size of F3 and randomly allocated into 4 groups $(n=14)$ according to the filling material and obturation technique; Group 1: gutta percha/cold lateral (GP/C), Group 2: Resilon/cold lateral (R/C), Group 3: gutta percha/thermal (GP/T), Group 4: Resilon/thermal (R/T). Thermal filling was performed using System B and Obtura II. The filled roots were then scanned using micro-CT and the data were subsequently processed using Avizo 6.3 Standard version software. Statistical analysis was performed using the Kruskal-Wallis test with multiple pairwise comparisons.

The total percentage volume of voids in GP/C (2.40\%) and R/C (1.88\%) were significantly higher than GP/T $(1.04 \%)$ and R/T $(1.29 \%)(<0.001)$. A significantly higher percentage of voids was present in the coronal third compared with middle and apical thirds $(P<0.001)$.

Thermal obturation resulted in a root filling with significantly fewer voids than the cold lateral condensation technique regardless of the obturation material used. Most of the voids located in the coronal third which emphasizes the importance of obtaining a good coronal seal following endodontic treatment.
\end{abstract}

Keywords: Computed tomography (CT); Resilon; voids.

Cite this Article as: Fatma Asheibi, Alison J.E. Qualtrough, Anthony Mellor, Philip J. Withers and Tristan Lowe (2014), "Micro-CT Evaluation of Voids in the Filling Material of Single-Rooted Teeth Obturated with Different Techniques," Journal of Research and Practice in Dentistry, Vol. 2014 (2014), Article ID 556901, DOI: $10.5171 / 2014.556901$ 


\section{Introduction}

Optimal obturation leads to successful endodontic treatment ( $\mathrm{Ng}$ et al. 2008). Incomplete filling of a well-cleaned and prepared root canal system could compromise the outcome of treatment $\mathrm{Ng}$ et al. 2008). The ideal obturation material should provide good adaptation to the canal walls and fill any irregularities with the full length of the canal being densely filled with a homogenous mass of obturation material (Schilder 1967).

Gutta percha (GP) has been the material of choice for obturation for many years as it possesses favourable properties such as dimensional stability, good working properties, ease of removal, biocompatibility and radiopacity (Miner $e t$ al. 2006). One of the limitations of GP is that it does not bond to any type of sealer (Teixeira et al. 2004a). As many sealers shrink upon setting, there is a potential for gaps or voids creation via which microleakage may occur (Shipper et al. 2004; Wedding et al. 2007).

A resin-based material, Resilon, was introduced in 2004 (Teixeira and Trope 2004). It has been claimed that it bonds to the root canal dentine, strengthens the root when used as an obturation material, (Teixeira et al. 2004b; Skidmore et al. 2006; Hammad et al. 2007; Monteiro et al. 2011) and provides a better seal than GP (Shipper et al. 2004; Wedding et al. 2007). It has similar handling properties as GP and can be placed into root canals using either cold lateral condensation (Hammad et al. 2009; Santos et al. 2010) or thermal obturation (Epley et al. 2006; Karabucak et al. 2008).

Many properties of Resilon have been investigated such as leakage resistance (Shipper et al. 2004), root strengthening (Teixeira et al. 2004b, Hammad et al. 2007) and quality of obturation (Hammad et al. 2009). However, most of these studies relate to it when used with cold lateral condensation and very few have compared it with thermal obturation (Epley et al. 2006). As the use of heated filling techniques is increasing (Lee et al. 2009), investigation of the quality of obturation with Resilon using this method in comparison with cold lateral condensation is timely.

The few studies which have investigated Resilon using thermal obturation in comparison with cold lateral condensation have used a variety of assessment methods which may result in inaccuracies. In one study (Epley et al. 2006), the filled roots were sectioned and examined under a stereomicroscope. This process may lead to loss of some of the filling material and creation of spaces that may appear as voids. In the present study, micro-CT was used as it gives highly accurate three-dimensional analysis of voids (Jung et al. 2005).

The aim of this study was to compare the percentage volume of voids in root canals filled with Resilon and with GP using cold lateral condensation and continuous wave of compaction techniques. The null hypothesis was that there would be no significant difference in the percentage volume of voids between the four combinations of the two materials and obturation techniques.

\section{Materials and Methods}

This study was approved by the ethics committee at the University of Manchester (Reference number: UREC5-10275). Seventy upper anterior human teeth were collected, disinfected with 5\% sodium hypochlorite (NaOCl) (Procter and Gamble, Weybridge Surrey, UK) for 10 minutes and stored in normal saline. Exclusion criteria were: roots that were carious, fractured, resorbed or immature. Roots with sclerosed canals, with more than one canal or those with root curvature $>10^{\circ}$, as determined by Schneider (1971), were also excluded.

\section{Preparation}

From the initial sample, a total of 56 teeth (28 lateral incisors, 16 central incisors and 12 canines) were selected according to the aforementioned criteria. The teeth were decoronated and the length of the roots was reduced to $14 \mathrm{~mm}$ using a diamond wheel saw with water cooling (central 
incisors, lateral incisors and canines were kept separate to facilitate in their allocation into groups for obturation). The working length was determined to be $1 \mathrm{~mm}$ short of the apical foramen using a size $10 \mathrm{~K}$-file (VDW GmbH, Munich, Germany).

The root canals were prepared using rotary ProTaper files to the final size of F3 (Dentsply Maillefer, Ballaigues, Switzerland) and irrigated with 1\% $\mathrm{NaOCl}$. After completion of the preparation, the root canals were irrigated with $5 \mathrm{~mL} \mathrm{17 \%}$ ethylene-diamine-tetra-acetic acid (EDTA) (PPH Cerkamed, Sandomierska, Poland) and $10 \mathrm{~mL}$ distilled water.

\section{Obturation of Root Canals}

The roots were randomly allocated into four groups using a stratified sampling method ( $n=14: 7$ lateral incisors, 4 central incisors and 3 canines) according to the filling material and obturation technique.

Group 1 (GP/C) was filled with ProTaper GP F3 (Dentsply Maillefer, Ballaigues, Switzerland) and AH-Plus sealer (Dentsply DeTrey GmbH, Konstanz, Germany) using cold lateral condensation. A master cone was selected and trimmed to fit the canal to the working length with tug-back. Then, the canal was dried using paper points (Diadent, Chungcheongbuk-do, Korea). The sealer was carried into the canal using the master cone. A finger spreader (Dentsply Maillefer, Ballaigues, Switzerland) was used to compact the GP cone and create a space for accessory points. When obturation was completed, the GP points were cut to the coronal end of the root using a heated instrument and the coronal part was compacted using a Machtou plugger (Dentsply Maillefer, Ballaigues, Switzerland).

Group 2 (R/C) was filled with Resilon/RealSeal (Pentron Corp., Wallingford, China) sealer using cold lateral condensation according to the manufacturers' instructions. A Resilon point (30/0.06) was used as the master cone which was trimmed to fit the canal to the working length with tug-back. After drying the root canal with paper points,
RealSeal primer was applied. RealSeal sealer was carried to the canal on the master cone. Cold lateral condensation was performed as previously described. The coronal surface was light cured for 40 seconds.

Group 3 (GP/T) was filled with ProTaper GP/AH-Plus sealer using a continuous wave of compaction technique. The sealer was carried to the canal using the master cone. A System B plugger of fine-medium size (SybronEndo, Glendora, USA) was selected and a rubber stopper was fitted $5 \mathrm{~mm}$ short of the working length, marking the binding point. The heat source was adjusted to $200^{\circ} \mathrm{C}$. The plugger was activated and used to cut the excess GP and then inserted into the canal along the master cone until the binding point was reached. The heat source was then deactivated and a firm pressure was maintained for 10 seconds. A onesecond heat burst was activated and the plugger was withdrawn. The canal was then backfilled with GP pellets (Obtura Spartan, Earth City, Missouri, USA) using Obtura III at $200^{\circ} \mathrm{C}$ (Obtura Spartan, Earth City, Missouri, USA).

Group 4 (R/T) was obturated with Resilon/RealSeal sealer using the continuous wave of compaction technique described above. The temperature setting in system B and Obtura III was set to $150^{\circ} \mathrm{C}$ according to the manufacturers' instructions.

All roots were stored for $72 \mathrm{hrs}$ at $37^{\circ} \mathrm{C}$ and $100 \%$ humidity in air-tight containers.

\section{Scanning of the Roots}

The roots were scanned in a random order using a $225 \mathrm{kV} 3 \mu \mathrm{m}$ source Nikon micro-CT housed in a walk-in enclosure (Nikon X-tek Systems Ltd., Tring, England). Using a sample-source distance of $2 \mathrm{~cm}$ and a detector-sample distance of $138 \mathrm{~cm}$, magnified images were collected on a 2000 pixel (200 micron pixel pitch) detector allowing observation of fine differences in contrast. The X-ray attenuation could be adjusted by changing the target material and accelerating voltage. For this scan, copper was used as the target material. The 
micro-CT settings were: $93 \mathrm{kV}, 135$ micro Amps, 1901 projections (radiographs), 1000 seconds exposure, gain 16 .

The raw data were reconstructed using CTPro 3D software (Version XT 2.2, Metris, Hertfordshire, UK). A effective voxel size of 7.7-9.3 $\mu \mathrm{m}$ was obtained. Voids were observed in 2-dimensional (2D) (Figure 1) and 3-dimensional (3D) images (Figure 2) using Avizo 6.3 Standard version
(Visualization Sciences Group, Berlin, Germany). A number of grey-scale thresholding values were tried and the upper and lower limit values demonstrated that the scatter per sample was found to be about $0.1 \%$ volume of voids. After segmentation, Avizo software was used to measure the total volume of voids. The percentage volume of voids was calculated using the following equation:

$$
\frac{\text { Total volume of voids }}{\text { Total volume of the canal }} \times 100
$$

\section{Statistical Analysis}

Test of normality showed that data were not normally distributed and therefore, non-parametric tests were used. Analysis of data was carried out using the KruskalWallis test with multiple pairwise comparisons. The software used was SPSS Version 20.0 (IBM Corp., Armonk, US) with the level of significance set at $5 \%$.

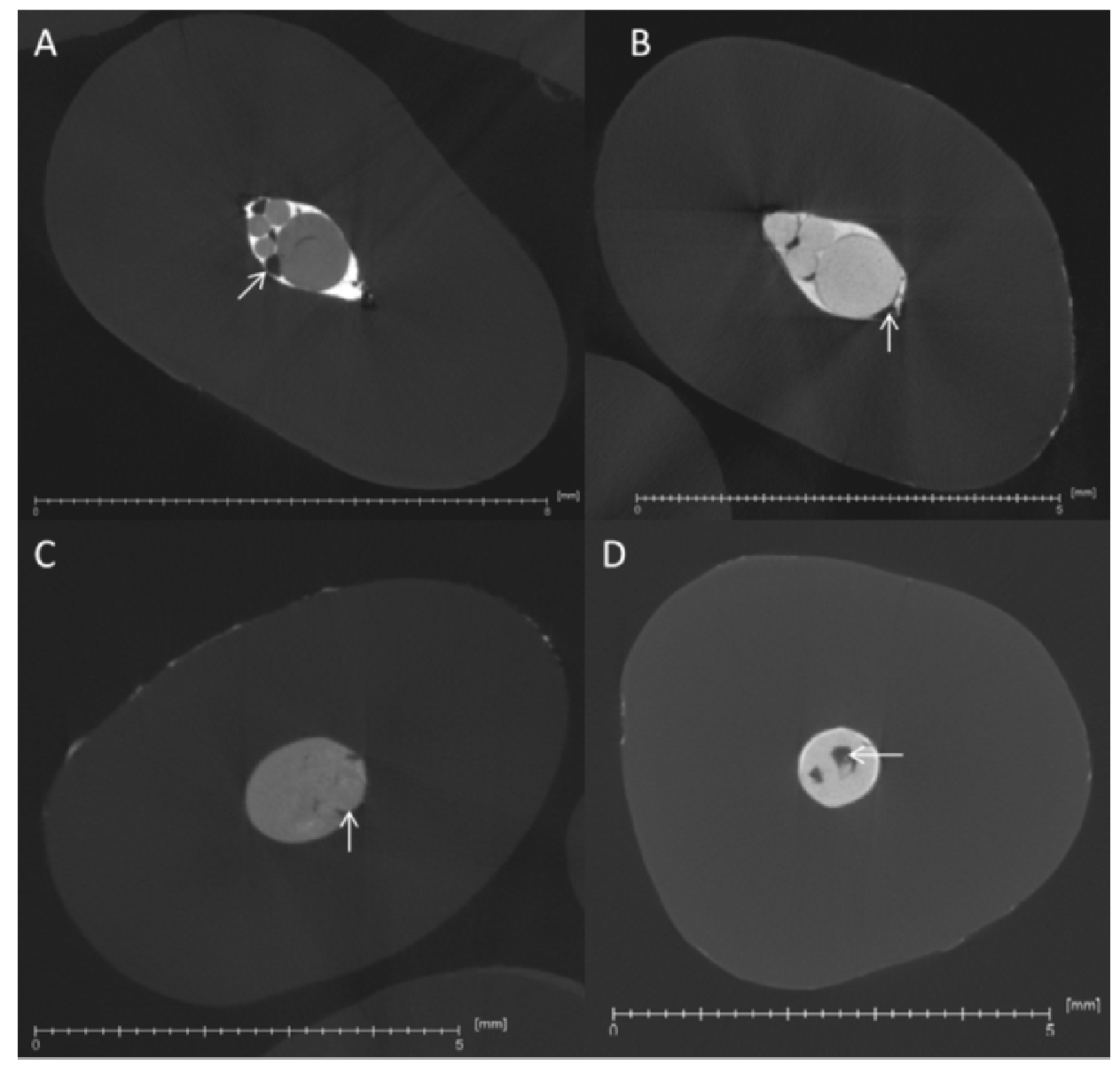

Figure 1. Visualization of Reconstructed Images Showing Virtual 2D Slices of the Filling (A) Gutta Percha/ Cold Lateral Condensation, (B) Resilon/Cold Lateral Condensation, (C) Gutta Percha/Thermal Compaction, (D) Resilon/Thermal Compaction. The Arrows Indicate Voids within the Filling Material and the Scale Bars are 5mm Long. 


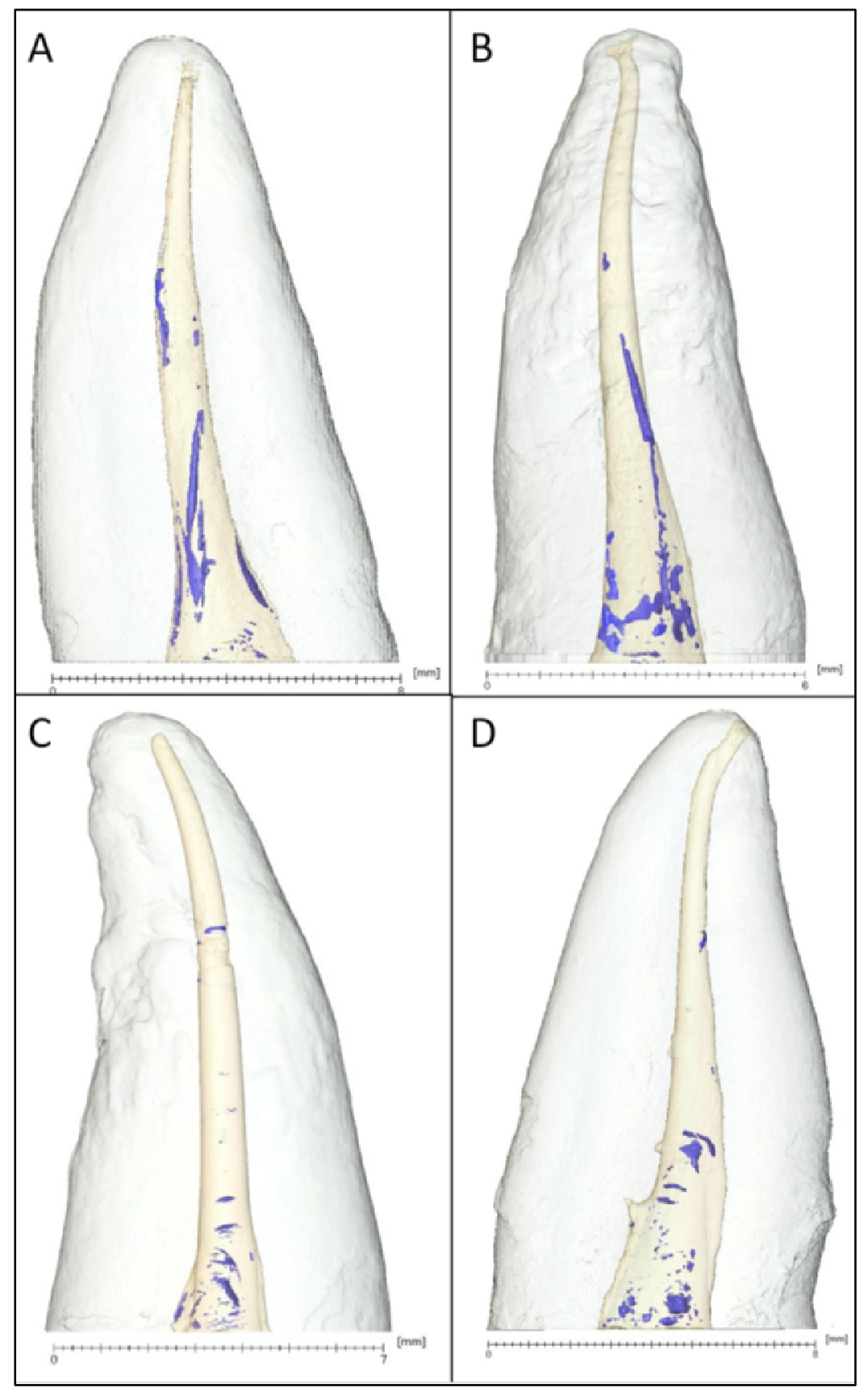

Figure 2. 3D Images of the Root Canal Fillings in (A) Gutta Percha/Cold Lateral Condensation, (B) Resilon/Cold Lateral Condensation, (C) Gutta Percha/Thermal Compaction, (D) Resilon/Thermal Compaction. The Blue Colour within the Filling Material Indicates Voids; the Scale Bars are 6-8mm Long.

\section{Results}

The means and standard deviations (SD) of the overall percentage (\%) of voids are shown in Table 1. The GP/C (gutta percha/cold lateral) group showed the highest overall mean value $(2.40 \%)$, whereas the GP/T (gutta percha/thermal) group had the lowest overall value (1.04\%). The Kruskal-Wallis test indicated that there was a statistically significant difference between the groups $(P<0.001)$. The multiple pairwise comparisons showed that Groups GP/C and R/C (Resilon/cold 
lateral) had significantly higher percentage of voids than Groups GP/T and R/T (Resilon/thermal) $(P<0.001$ for $\mathrm{GP} / \mathrm{C}$ versus $\mathrm{GP} / \mathrm{T}$ and $P=0.007$ for $\mathrm{R} / \mathrm{C}$ versus $\mathrm{R} / \mathrm{T})$.

Table 2 shows the means and standard deviations of the percentage of voids in each third of the root canal. In the coronal and the middle thirds, the results were similar to those for the overall percentage of voids (GP/C and R/C showed significantly higher percentage of voids than GP/T and $\mathrm{R} / \mathrm{T})(P<0.05)$. In the apical third, there was no significant difference between the groups. When the thirds were compared for each group, the coronal third showed significantly higher percentage of voids than the middle third, which had significantly higher percentage of voids than the apical third $(P<0.001)$. The percentage of voids in the coronal third in relation with the overall percentage of voids was $68.8 \%$ in $\mathrm{GP} / \mathrm{C}, 75.5 \%$ in $\mathrm{R} / \mathrm{C}$, $70.2 \%$ in $\mathrm{G} / \mathrm{T}$ and $73.7 \%$ in $\mathrm{R} / \mathrm{T}$.

Table 1. Means and Standard Deviations of the Overall Percentage of Voids

\begin{tabular}{|c|c|}
\hline $\begin{array}{c}\text { Group } \\
\text { (n=14) }\end{array}$ & $\begin{array}{c}\text { Overall voids \% mean } \\
\text { (SD) }\end{array}$ \\
\hline GP/C & $2.40^{\mathrm{a}}(0.66)$ \\
\hline R/C & $1.88^{\mathrm{a}}(0.61)$ \\
\hline GP/T & $1.04^{\mathrm{b}}(0.44)$ \\
\hline R/T & $1.29^{\mathrm{b}}(0.44)$ \\
\hline
\end{tabular}

GP/C: gutta percha/ cold lateral condensation

$\mathrm{R} / \mathrm{C}$ : Resilon/cold lateral condensation

GP/T: gutta percha/thermal compaction

$\mathrm{R} / \mathrm{T}$ : Resilon/thermal compaction

Different superscript letters indicate statistically significant differences

Table 2. Means and Standard Deviations of the Percentage of Voids in Each Third of the Root Canal

\begin{tabular}{|c|c|c|c|}
\hline $\begin{array}{c}\text { Group } \\
\text { (n=14) }\end{array}$ & $\begin{array}{c}\text { Coronal voids \% } \\
\text { mean (SD) }\end{array}$ & $\begin{array}{c}\text { Middle voids \% } \\
\text { mean (SD) }\end{array}$ & $\begin{array}{c}\text { Apical voids \% } \\
\text { mean (SD) }\end{array}$ \\
\hline GP/C & $1.65(0.60)$ & $0.66(0.22)$ & $0.09(0.05)$ \\
\hline R/C & $1.42(0.57)$ & $0.44(0.21)$ & $0.02(0.01)$ \\
\hline GP/T & $0.73(0.30)$ & $0.27(0.20)$ & $0.04(0.03)$ \\
\hline R/T & $0.95(0.40)$ & $0.26(0.12)$ & $0.08(0.06)$ \\
\hline
\end{tabular}

GP/C: gutta percha/ cold lateral condensation

$\mathrm{R} / \mathrm{C}$ : Resilon/cold lateral condensation

GP/T: gutta percha/thermal compaction

$\mathrm{R} / \mathrm{T}$ : Resilon/thermal compaction

The differences between the thirds in all groups are statistically significant. GP/C and R/C have significantly higher volume of voids than GP/T and R/T in the coronal and middle thirds.

\section{Discussion}

Traditional methods used to evaluate the quality of root fillings allow only partial evaluation of the root canal content. Radiographs give a two-dimensional view of a three-dimensional structure. Sectioning the roots to view them using a scanning electron microscope or a stereomicroscope could result in loss of some of the material which may mimic voids. Further, the chance of intersecting a void is statistical and the shape of the voids may mean that volume fractions are not properly inferred from 2D sections. The clearing technique is time consuming (Anbu et al. 2010) and the long periods of immersion in alcohol may affect the physical properties of gutta percha (Moller and Orstavik 1985). Recently, the use of 
computed tomography (CT) (Barletta et al. 2008; Kandaswamy et al. 2009; Anbu et al. 2010) and micro-CT (Zakizadeh et al. 2008; Roggendorf et al. 2010; Hatamleh and Watts 2011) in dental research has gained increasing popularity. It gives an accurate three-dimensional view of the root canal content without destroying the samples which can be used for further research (Hammad et al. 2009). In the present study, the voxel size obtained was 7.7-9.3 $\mu \mathrm{m}$, which is better than that used in previously published studies (Hammad et al. 2009; Somma et al. 2011).

The null hypothesis was rejected as the results showed a statistically significant difference in the volume of voids between the tested groups. The cold lateral condensation technique with both materials showed a significantly higher percentage of voids in comparison with the thermal technique. This agrees with previous studies (Kandaswamy et al. 2009; Anbu et al. 2010; Peters et al. 2010) which compared the two techniques using gutta percha. In cold lateral condensation, the resulting mass is not homogenous and there may be spaces present between the points. By contrast, the thermal technique produces a more homogenous mass with fewer voids and better adaptation of the filling to the canal walls (Anbu et al. 2010; Peters et al. 2010). However, during condensation with pluggers, air could be entrapped. None of our samples were voidfree. This finding has also been reported in previous studies (Epley et al. 2006; Gulsahi et al. 2007; James et al. 2007).

Our study showed that the GP/C group had a higher percentage of voids than the $\mathrm{R} / \mathrm{C}$ group, but this was not statistically significant. This is consistent with other studies (Gulsahi et al. 2007; James et al. 2007; Akman et al. 2010), but is in contrast with that found by Hammad et al. (2009). In the latter study (Hammad et al. 2009), the lowest percentage of voids was found in the GP group as compared with Resilon, GuttaFlow and EndoRez. The sealer used with GP was Tubliseal (a zinc-oxide eugenol-based sealer), whereas in the present study AH-Plus sealer (a resinbased sealer) was used. It has been reported that GP showed volumetric expansion when it came into contact with eugenol which resulted in a better seal of the obturation (Michaud et al. 2008). In addition, the voxel size used in the present study was lower than that used in the study by Hammad et al. (2009) which improved detection of smaller voids. This may explain the fewer voids in the GP group reported by Hammad et al. (2009).

In contrast with the findings of Hammad et al. (2009), the results of the present study showed that the coronal third in all groups contained a significantly higher percentage of voids than the middle and apical thirds. This may have a clinical significance if the coronal seal lost as it may accelerate penetration of fluids through the root canal filling and dissolution of the sealer. In both obturation techniques used in this study, the apical third of the canal was filled with a master cone. In the cold lateral condensation technique, the remainder of the canal was filled with accessory points which were packed using a spreader leading to a greater risk of void creation between the accessory points in the middle and coronal thirds as opposed to the apical third. Similarly, in the thermal obturation technique, the middle and coronal thirds were obturated with a softened filling material in which air bubbles may be entrapped during condensation. This may explain the significant difference in the percentage of voids between the thirds.

Voids inside the filling materials, which are not linked to the periphery of the filling, could be considered less clinically significant than peripheral gaps because micro-organisms, if present, are restrained in an unfavourable environment and do not have access to a nutritional supply. Peripheral gaps along the dentine-sealer or core material-sealer interfaces may jeopardize the outcome of root canal treatment ( $\mathrm{Ng}$ et al. 2008). This is because they may act as a pathway that permits sealer dissolution and passage of microorganisms through the filled root canal to the peri-radicular tissues. Furthermore, if residual micro-organisms remain trapped in the dentinal tubules after treatment is finished, the peripheral gaps may form 
channels through which the microorganisms can get access to nutrients and initiate or continue the inflammatory process.

This is an in vitro study and further in vivo investigations are indicated. Variations in the canal anatomy of the human teeth may affect the obturation quality. Ideally, contralateral teeth from the same individual should be used (De-Deus 2012). However, this is not practical in research settings and limits the sample size. Therefore, upper anterior teeth were used in this study and each group contained equal number of central incisors, lateral incisors and canines (stratified sampling). It would be beneficial in the future to extend the testing to a wider range of root curvatures as this would be more clinically relevant.

\section{Conclusion}

The results of the present study show that the percentage of voids in the fillings is low $(1.04 \%-2.40 \%)$, regardless of the filling technique. However, the significant difference shown highlights the importance of the use of continuous wave of compaction technique in reducing the volume of voids in the root canal filling. In addition, the significantly higher percentage of voids in the coronal third in comparison with the middle and the apical thirds emphasizes the significance of obtaining a good coronal seal after completion of endodontic treatment.

\section{Acknowledgement}

The authors deny any conflicts of interest. We are grateful to the EPSRC whose grant funding (EP/I02249X, EP/F007906 and EP/F028431) enabled the purchase and support of the imaging equipment in the Henry Moseley X-ray Imaging Facility.

\section{References}

Akman, M., Akman, S., Derinbay, O. \& Belli, S. (2010). "Evaluation of Gaps or Voids Occurring in Roots Filled with Three Different Sealers," European Journal of Dentistry, 4 (2) 101-109.
Anbu, R., Nandini, S. \& Velmurugan, N. (2010). "Volumetric Analysis of Root Fillings Using Spiral Computed Tomography: An in Vitro Study," International Endodontic Journal, 43 (1) 6468.

Barletta, F. B., de Sousa Reis, M., Wagner, M., Borges, J. C. \& Dall'Agnol, C. (2008). "Computed Tomography Assessment of Three Techniques for Removal of Filling Material," Australian Endodontic Journal, 34 (3) 101-105.

De-Deus, G. (2012). "Research that Matters - Root Canal Filling and Leakage Studies," International Endodontic Journal, 45 (12) 1063-1064.

Epley, S. R., Fleischman, J., Hartwell, G. \& Cicalese, C. (2006). "Completeness of Root Canal Obturations: Epiphany Techniques versus Gutta-Percha Techniques," Journal of Endodontics, 32 (6) 541-544.

Gulsahi, K., Cehreli, Z. C., Onay, E. O., Tasman-Dagli, F. \& Ungor, M. (2007). "Comparison of the Area of Resin-Based Sealer and Voids in Roots Obturated with Resilon and Gutta-Percha," Journal of Endodontics, 33 (11) 1338-1341.

Hammad, M., Qualtrough, A. \& Silikas, N. (2007). "Effect of New Obturating Materials on Vertical Root Fracture Resistance of Endodontically Treated Teeth," Journal of Endodontics, 33 (6) 732-736.

Hammad, M., Qualtrough, A. \& Silikas, N. (2009). "Evaluation of Root Canal Obturation: A Three-Dimensional in Vitro Study," Journal of Endodontics, 35 (4) 541544.

Hatamleh, M. M. \& Watts, D. C. (2011). "Porosity and Color of Maxillofacial Silicone Elastomer," Journal of Prosthodontics, 20 (1) 60-66.

James, B. L., Brown, C. E., Legan, J. J., Moore, B. K. \& Vail, M. M. (2007). "An in Vitro Evaluation of the Contents of Root Canals Obturated with Gutta Percha and AH-26 Sealer or Resilon and Epiphany Sealer," Journal of Endodontics, 33 (11) 1359-1363. 
Jung, M., Lommel, D. \& Klimek, J. (2005). "The Imaging of Root Canal Obturation Using Micro CT," International Endodontic Journal, 38 (9) 617-626.

Kandaswamy, D., Venkateshbabu, N., Reddy, G. K., Hannah, R., Arathi, G. \& Roohi, R. (2009). "Comparison of Laterally Condensed, Vertically Compacted Thermoplasticized, Cold Free-Flow GP Obturations-A Volumetric Analysis Using Spiral CT," Journal of Conservative Dentistry, 12 (4) 145-149.

Karabucak, B., Kim, A., Chen, V. \& Iqbal, M. K. (2008). "The Comparison of GuttaPercha and Resilon Penetration into Lateral Canals with Different Thermoplastic Delivery Systems," Journal of Endodontics, 34 (7) 847-849.

Lee, M., Winkler, J., Hartwell, G., Stewart, J. \& Caine, R. (2009). "Current Trends in Endodontic Practice: Emergency Treatments and Technological Armamentarium," Journal of Endodontics, 35 (1) 35-39.

Michaud, R. A., Burgess, J., Barfield, R. D., Cakir, D., McNeal, S. F. \& Eleazer, P. D. (2008). "Volumetric Expansion of GuttaPercha in Contact with Eugenol," Journal of Endodontics, 34 (12) 1528-1532.

Miner, M. R., Berzins, D. W. \& Bahcall, J. K. (2006). "A Comparison of Thermal Properties between Gutta-Percha and a Synthetic Polymer Based Root Canal Filling Material (Resilon)," Journal of Endodontics, 32 (7) 683-686.

Moller, B. \& Orstavik, D. (1985). "Influence of Antiseptic Storage Solutions on Physical Properties of Endodontic Guttapercha Points," Scandinavian Journal of Dental Research, 93 (2) 158-161.

Monteiro, J., de Ataide, I. N., Chalakkal, P. \& Chandra, P. K. (2011). "In Vitro Resistance to Fracture of Roots Obturated with Resilon or Gutta-Percha," Journal of Endodontics, 37 (6) 828-831.

Ng, Y. L., Mann, V., Rahbaran, S., Lewsey, J. \& Gulabivala, K. (2008). "Outcome of Primary Root Canal Treatment: Systematic Review of the Literature-Part 2. Influence of Clinical Factors," International Endodontic Journal, 41 (1) 6-31.

Peters, C. I., Sonntag, D. \& Peters, O. A. (2010). "Homogeneity of Root Canal Fillings Performed by Undergraduate Students with Warm Vertical and Cold Lateral Techniques," Oral Surgery Oral Medicine Oral Pathology Oral Radiology and Endodontology, 110 (3) E41-E49.

Roggendorf, M., Legner, M., Ebert, J., Fillery, E., Frankenberger, R. \& Friedman, S. (2010). "Micro-CT Evaluation of Residual Material in Canals Filled with Activ GP or GuttaFlow Following Removal with NiTi Instruments," International Endodontic Journal, 43 (3) 200-209.

Santos, J., Tjaderhane, L., Ferraz, C., Zaia, A., Alves, M., De Goes, M. \& Carrilho, M. (2010). "Long-Term Sealing Ability of Resin-Based Root Canal Fillings," International Endodontic Journal, 43 (6) 455-460.

Schilder, H. (1967). "Filling Root Canals in Three Dimensions," Dental Clinics of North America, 11 (4) 723-744.

Schneider, S. W. (1971). "A Comparison of Canal Preparations in Straight and Curved Root Canals," Oral Surgery, Oral Medicine, Oral Pathology, 32 (2) 271-275.

Shipper, G., Ørstavik, D., Teixeira, F. B. \& Trope, M. (2004). "An Evaluation of Microbial Leakage in Roots Filled with a Thermoplastic Synthetic Polymer-Based Root Canal Filling Material (Resilon)," Journal of Endodontics, 30 (5) 342-347.

Skidmore, L. J., Berzins, D. W. \& Bahcall, J. K. (2006). "An in Vitro Comparison of the Intraradicular Dentin Bond Strength of Resilon and Gutta-Percha," Journal of Endodontics, 32 (10) 963-966.

Somma, F., Cretella, G., Carotenuto, M., Pecci, R., Bedini, R., De Biasi, M. \& Angerame, D. (2011). "Quality of Thermoplasticized and Single Point Root Fillings Assessed by Micro Computed Tomography," International Endodontic Journal, 44 (4) 362-369. 
Teixeira, F. B., Teixeira, E. C. N., Thompson, J. Y., Leinfelder, K. F. \& Trope, M. (2004a). "Dentinal Bonding Reaches the Root Canal System," Journal of Esthetic and Restorative Dentistry, 16 (6) 348-354.

Teixeira, F. B., Teixeira, E. C. N., Thompson, J. Y. \& Trope, M. (2004b). "Fracture Resistance of Roots Endodontically Treated with a New Resin Filling Material," The Journal of the American Dental Association, 135 (5) 646-652.

Teixeira, F. B. \& Trope, M. (2004). "GuttaPercha--The End of an Era?," Alpha Omegan, 97 (4) 16-22.
Wedding, J. R., Brown, C. E., Legan, J. J., Moore, B. K. \& Vail, M. M. (2007). "An in Vitro Comparison of Microleakage between Resilon and Gutta-Percha with a Fluid Filtration Model," Journal of Endodontics, 33 (12) 1447-1449.

Zakizadeh, P., Marshall, S. J., Hoover, C. I., Peters, O. A., Noblett, W. C., Gansky, S. A. \& Goodis, H. E. (2008). "A Novel Approach in Assessment of Coronal Leakage of Intraorifice Barriers: A Saliva Leakage and Micro-Computed Tomographic Evaluation," Journal of Endodontics, 34 (7) 871-875. 